\title{
The anti-cancer components of Ganoderma lucidum possesses cardiovascular protective effect by regulating circular RNA expression
}

\author{
Yi-Zhen Xie ${ }^{1,2, *}$, Fenghua Yang ${ }^{3, *}$, Weijiang Tan $^{3}$, Xiangmin Li ${ }^{1,4}$, Chunwei Jiao ${ }^{2}$, \\ Ren Huang ${ }^{3}$, Burton B. Yang ${ }^{4,5}$ \\ ${ }^{1}$ State Key Laboratory of Applied Microbiology Southern China, Guangdong Institute of Microbiology, Guangzhou, China \\ ${ }^{2}$ Guangdong Yuewei Edible Fungi Technology Co. Ltd., Guangzhou, China \\ ${ }^{3}$ Guangdong Laboratory Animals Monitoring Institute, Guangdong Provincial Key Laboratory of Laboratory Animals, \\ Guangzhou, Guangdong, China \\ ${ }^{4}$ Sunnybrook Research Institute, Sunnybrook Health Sciences Centre, Toronto, ON, Canada \\ ${ }^{5}$ Department of Laboratory Medicine and Pathobiology, University of Toronto, Toronto, ON, Canada \\ * These authors contributed equally \\ Correspondence to: Burton B. Yang, email: byang@sri.utoronto.ca
}

Keywords: herbal medicine, Ganoderma lucidum, anti-cancer, heart function, circular RNA

Received: June 14,2016 Accepted: August 12, $2016 \quad$ Published: August 28, 2016

This is an open-access article distributed under the terms of the Creative Commons Attribution License, which permits unrestricted use, distribution, and reproduction in any medium, provided the original author and source are credited.

\section{ABSTRACT}

To examine the role of oral Ganoderma spore oil in cardiovascular disease, we used transverse aortic constriction (TAC) in mice to model pressure overload-induced cardiomyopathy. Our preliminary results demonstrated a potential cardioprotective role for spore oil extracted from Ganoderma. We found that Ganoderma treatment normalized ejection fraction and corrected the fractional shortening generated by TAC. We also found evidence of reduced left ventricular hypertrophy as assessed by left ventricular end diastolic diameter. Analysis of total RNA expression using cardiac tissue samples from these mice corroborated our findings. We found reduced expression of genes associated with heart failure, including a novel circular RNA circ-Fox03. Thus our data provides evidence for Ganoderma lucidum as a potential cardioprotective agent, warranting further preclinical exploration.

\section{INTRODUCTION}

Complementary and alternative medicines have attracted increasing attention as disease treatments, adjuvants, and alternative supplements [1-4]. Medicinal mushrooms comprise a large proportion of these alternative products, among which Ganoderma lucidum is the most highly studied [5-8]. Preclinical studies have demonstrated anti-tumorigenic roles in a range of medicinal mushrooms [9-11]. A Cochrane metaanalysis showed that patients who had been administered Ganoderma lucidum alongside chemo/radiotherapy were more likely to respond positively compared to chemo/radiotherapy alone. These trials demonstrated improved immune functions as measured by increased CD3, CD4, and CD8 immune response cells [12]. In vitro, Ganoderma lucidum was also found to inhibit proliferation and induce apoptosis in ovarian, colon, and gastric cancer cell lines [13-15]. Ganoderma lucidum contains beta glucans and other polysaccharides which stimulate innate immunity and activate host dendritic cells $[16,17]$. Ganoderma lucidum also produces a group of ganoderic acids, which have molecular structures that are similar to steroids $[18,19]$.

\section{DISCUSSION}

An exploratory trial of 26 patients with hypertension and/or dyslipidemia demonstrated that Ganoderma lucidum treatment reduced total triglycerides and increased HDL-cholesterol, implicating a cardio-protective role [20]. We employed a transverse aortic constriction (TAC) mouse model of pressure overload-induced cardiomyopathy and heart failure to examine the role of 
Ganoderma spore oil administration. The TAC model induces an initial compensatory cardiac remodeling which enhances cardiac contractility. Gradually, the response to chronic overload leads to cardiac dilatation and heart failure. The murine TAC model has been extensively used to study cardiovascular disease and to elucidate signaling pathways involved in cardiac hypertrophy and heart failure.

TAC mice were administered oral Ganoderma spore oil every other day for 14 days. The control group were administered vegetable oil and an anti-hypertensive $\beta 2$ adrenergic receptor antagonist. Mice were anesthetized with $2 \%$ isoflurane inhalation for transthoracic echocardiography and invasive hemodynamic assessment. Transthoracic echocardiography was performed to measure left ventricular ejection fraction (LVEF), left ventricular fractional shortening (LVFS), left ventricular end diastolic diameter, and cardiac output. Data analysis was conducted in a blinded manner.

Relative to the $65.23 \%$ ejection fraction in healthy sham mice, TAC mice were found to have a $43.26 \%$ ejection fraction (Fig 1A). This was below the normal range of $55-75 \%$, validating the TAC model we employed. Treatment with the anti-hypertensive medication approximated the normal range, while delivery of Ganoderma spore oil recovered the stroke volume to normal ranges. Consistent with these results, TAC mice displayed 21.7\% fractional shortening (Fig 1B). This was within the mildly abnormal range of $20-25 \%$, while treatment with Ganoderma spore oil brought it to the normal range of $25-45 \%$. The TAC mice also showed increased left ventricular end diastolic diameters, while mice treated with Ganoderma spore oil did not exhibit the same levels of left ventricular hypertrophy (Fig 2A). As a result, mice treated with Ganoderma spore oil recovered to physiologic cardiac output levels at $24.1 \mathrm{ml} / \mathrm{min}$ (Fig 2B). This led to improved vascular perfusion within the mice (Fig 2B). This suggests that Ganoderma spore oil increases the heart function to meet the demands of the body.

After functional analyses, mice were sacrificed and heart tissue was harvested. Total RNA was extracted with an RNeasy Mini Kit (Qiagen), followed by real-time PCR measurement with miScriptSYBR GreenPCR Kit (Qiagen) as described [21] to analyze levels of a circular RNA circ-
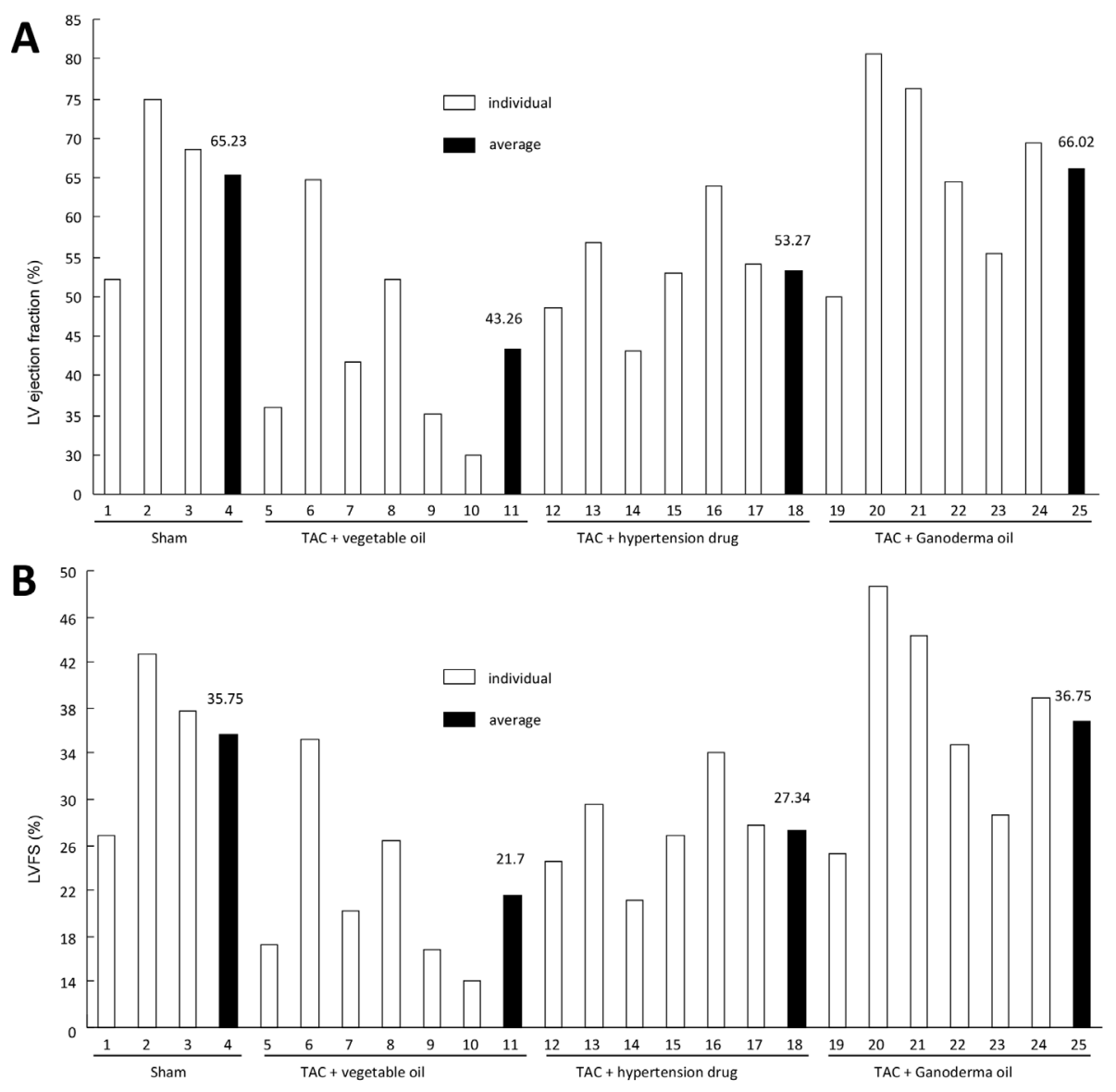

Fig 1. Ganoderma spore oil improves ejection fraction and fractional shortening. TAC mice showed decreased levels of LVEF (A) and LVFS (B). Treatment with Ganoderma spore oil increased the levels of LVEF and LVFS. 
Foxo3. We recently demonstrated that expression of circFoxo3 RNA could inhibit tumor cell cycle progression $[22,23]$ and promote cardiac senescence [24]. In this study, mice injected with the chemotherapeutic agent Doxorubicin (Dox) for induction of cardiomyopathy were analyzed for RNA expression levels. Mice with reduced cardiac function had increased expression of circ-Foxo3 RNA [24]. In the current study, we found that treatment with Ganoderma spore oil decreased levels of circ-Foxo3 (Fig 3A).

To corroborate these results, we cultured mouse cardiac fibroblasts and treated the cells with Ganoderma spore oil, following hydrogen peroxide induced oxidative stress. Relative to control groups, treatment with Ganoderma spore oil decreased circ-Foxo3 levels in a concentration- (Fig 3B) and time-dependent manner (Fig 3C).

Our previous studies have shown that the oil fraction of Ganoderma spores could induce death in versican-transformed cancer cells [25]. Further study found that the Ganoderma spore oil could induce death of cancer stem-like cells [6], potentially mediated by the molecule ergosterol peroxide [19]. Our preclinical results here show that Ganoderma spore oil has a protective role within the cardiovascular system. Treatment in TAC mice was found to normalize ejection fraction and correct the fractional shortening generated by this model. We also found evidence of reduced left ventricular hypertrophy as assessed by left ventricular end diastolic diameter. Thus in our TAC model, cardiac output was improved by oral administration of Ganoderma spore oil. This data provides rationale for further preclinical exploration of Ganoderma lucidum as a cardioprotective agent.

\section{ACKNOWLEDGEMENTS}

This work was supported by the High-Level Leading Talent Introduction Program of GDAS (2016GDASRC-0102), The Introduction of Leading Talent Project of Guangdong Province, and Open Fund of the Guangdong Provincial Key Laboratory of Laboratory Animals (No. 2013A061402001).
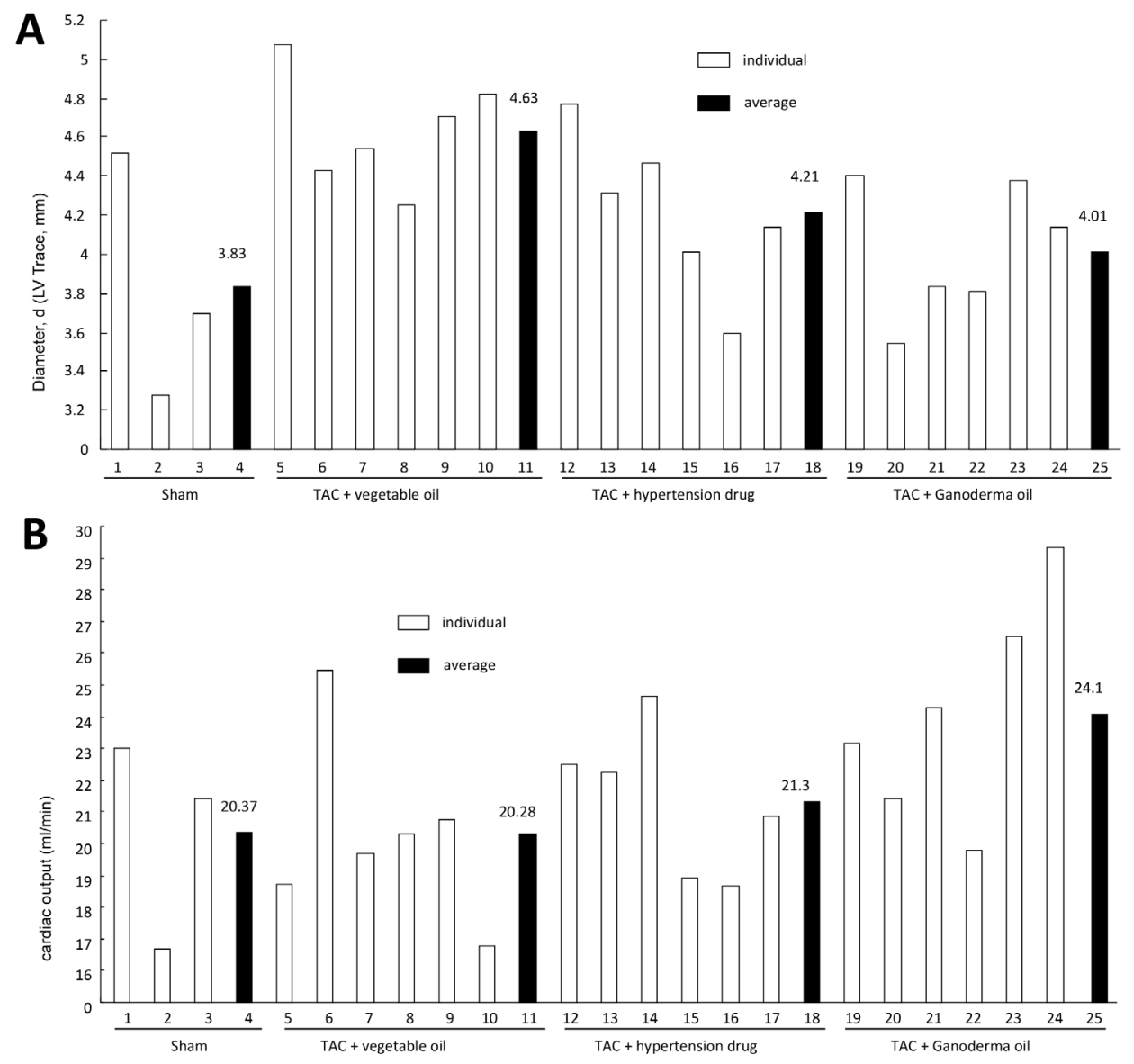

Fig 2. Ganoderma spore oil improves cardiac output. (A) TAC mice had increased left ventricular end diastolic diameters, while treatment with Ganoderma spore oil decreased the end diastolic diameters. (B) Treatment with Ganoderma spore oil increased cardiac output. 

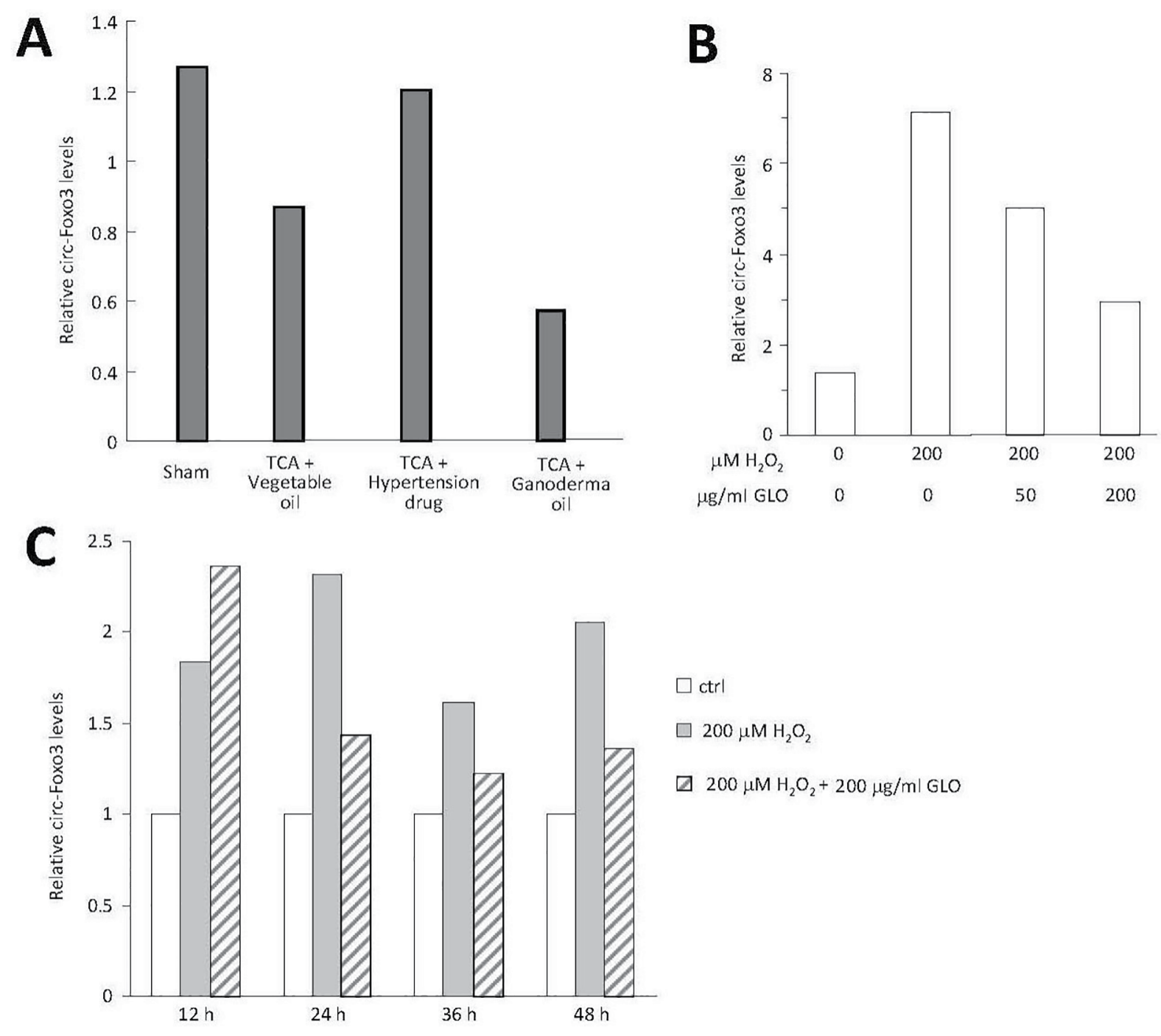

Fig 3. Treatment with Ganoderma spore oil decreases expression of circ-Foxo3. (A) Mice orally delivered with Ganoderma spore oil expressed decreased levels of circ-Foxo3 in the heart tissues. (B) Mouse cardiac fibroblasts treated with different concentrations of Ganoderma spore oil (GSO) expressed decreased levels of circ-Foxo3 in a concentration-dependent manner. (C) Mouse cardiac fibroblasts treated with Ganoderma spore oil expressed lower levels of circ-Foxo3 than the controls, which was time-dependent.

\section{CONFLICTS OF INTEREST}

No conflicts of interest were disclosed.

\section{REFERENCES}

1. Gao G, Chen L, Li J, Zhang D, Fang Y, Huang H, Chen $\mathrm{X}$ and Huang $\mathrm{C}$. Isorhapontigenin (ISO) inhibited cell transformation by inducing $\mathrm{G} 0 / \mathrm{G} 1$ phase arrest via increasing MKP-1 mRNA Stability. Oncotarget. 2014; 5(9):2664-2677. doi: 10.18632/oncotarget.1872.

2. Pozarowski P, Halicka DH and Darzynkiewicz Z. Cell cycle effects and caspase-dependent and independent death of HL-60 and Jurkat cells treated with the inhibitor of NF-
kappaB parthenolide. Cell Cycle. 2003; 2(4):377-383.

3. Ryu E, Son M, Lee M, Lee K, Cho JY, Cho S, Lee SK, Lee YM, Cho H, Sung GH and Kang H. Cordycepin is a novel chemical suppressor of Epstein-Barr virus replication. Oncoscience. 2014; 1(12):866-881. doi: 10.18632/ oncoscience.110/

4. Liu T, Men Q, Wu G, Yu C, Huang Z, Liu X and Li W. Tetrandrine induces autophagy and differentiation by activating ROS and Notch1 signaling in leukemia cells. Oncotarget. 2015; 6(10):7992-8006. doi: 10.18632/ oncotarget.3505.

5. Liu DL, Li YJ, Yang DH, Wang CR, Xu J, Yao N, Zhang XQ, Chen ZS, Ye WC and Zhang DM. Ganoderma lucidum derived ganoderenic acid $\mathrm{B}$ reverses $\mathrm{ABCB} 1-$-mediated multidrug resistance in HepG2/ADM cells. Int $\mathrm{J}$ Oncol. 2015. 
6. Wu QP, Xie YZ, Deng Z, Li XM, Yang W, Jiao CW, Fang L, Li SZ, Pan HH, Yee AJ, Lee DY, Li C, Zhang Z, Guo J and Yang BB. Ergosterol peroxide isolated from Ganoderma lucidum abolishes microRNA miR-378-mediated tumor cells on chemoresistance. PLoS One. 2012; 7(8):e44579.

7. Zhao S, Ye G, Fu G, Cheng JX, Yang BB and Peng C. Ganoderma lucidum exerts anti-tumor effects on ovarian cancer cells and enhances their sensitivity to cisplatin. Int J Oncol. 2011; 38(5):1319-1327.

8. Xie YZ LS, Yee A, La Pierre DP, Deng Z, Lee DY, Wu QP, Chen Q, Li C, Zhang Z, Guo J, Jiang Z, and Yang BB. Ganoderma lucidum inhibits tumour cell proliferation and induces tumour cell death. Enzyme \& Microbial Technol. 2006; 40:177-185.

9. Jiao C, Xie YZ, Yang X, Li H, Li XM, Pan HH, Cai $\mathrm{MH}$, Zhong HM and Yang BB. Anticancer activity of Amauroderma rude. PLoS One. 2013; 8(6):e66504.

10. Pan H, Han Y, Huang J, Yu X, Jiao C, Yang X, Dhaliwal $\mathrm{P}, \mathrm{Xie} \mathrm{Y}$ and Yang BB. Purification and identification of a polysaccharide from medicinal mushroom Amauroderma rude with immunomodulatory activity and inhibitory effect on tumor growth. Oncotarget. 2015; 6(19):17777-17791. doi: 10.18632/oncotarget.4397.

11. Li X, Wu Q, Xie Y, Ding Y, Du WW, Sdiri M and Yang BB. Ergosterol purified from medicinal mushroom Amauroderma rude inhibits cancer growth in vitro and in vivo by up-regulating multiple tumor suppressors. Oncotarget. 2015; 6(19):17832-17846. doi: 10.18632/ oncotarget.4026.

12. Jin X, Ruiz Beguerie J, Sze DM and Chan GC. Ganoderma lucidum (Reishi mushroom) for cancer treatment. Cochrane Database Syst Rev. 2016; 4:CD007731.

13. Dai S, Liu J, Sun X and Wang N. Ganoderma lucidum inhibits proliferation of human ovarian cancer cells by suppressing VEGF expression and up-regulating the expression of connexin 43. BMC Complement Altern Med. 2014; 14:434.

14. Liang Z, Guo YT, Yi YJ, Wang RC, Hu QL and Xiong XY. Ganoderma lucidum polysaccharides target a Fas/caspase dependent pathway to induce apoptosis in human colon cancer cells. Asian Pac J Cancer Prev. 2014; 15(9):39813986.

15. Jang KJ, Han MH, Lee BH, Kim BW, Kim CH, Yoon HM and Choi YH. Induction of apoptosis by ethanol extracts of Ganoderma lucidum in human gastric carcinoma cells. J Acupunct Meridian Stud. 2010; 3(1):24-31.

16. Hsu HY, Hua KF, Wu WC, Hsu J, Weng ST, Lin TL, Liu CY, Hseu RS and Huang CT. Reishi immuno-modulation protein induces interleukin-2 expression via protein kinasedependent signaling pathways within human T cells. J Cell Physiol. 2008; 215(1):15-26.

17. Lin YL, Liang YC, Lee SS and Chiang BL. Polysaccharide purified from Ganoderma lucidum induced activation and maturation of human monocyte-derived dendritic cells by the NF-kappaB and p38 mitogen-activated protein kinase pathways. J Leukoc Biol. 2005; 78(2):533-543.

18. Paterson RR. Ganoderma - a therapeutic fungal biofactory. Phytochemistry. 2006; 67(18):1985-2001.

19. Li X, Wu Q, Bu M, Hu L, Du WW, Jiao C, Pan H, Sdiri M, Wu N, Xie Y and Yang BB. Ergosterol peroxide activates Foxo3a-mediated cell death signaling by inhibiting AKT and c-Myc in human hepatocellular carcinoma cells. Oncotarget. 2016. doi: 10.18632/oncotarget.8608.

20. Chu TT, Benzie IF, Lam CW, Fok BS, Lee KK and Tomlinson B. Study of potential cardioprotective effects of Ganoderma lucidum (Lingzhi): results of a controlled human intervention trial. Br J Nutr. 2012; 107(7):10171027.

21. Li H, Gupta S, Du WW and Yang BB. MicroRNA-17 inhibits tumor growth by stimulating T-cell mediated host immune response. Oncoscience. 2014; 1(7):531-539. doi: 10.18632/oncoscience.69.

22. Du WW, Yang W, Liu E, Yang Z, Dhaliwal P and Yang BB. Foxo3 circular RNA retards cell cycle progression via forming ternary complexes with p21 and CDK2. Nucleic Acids Res. 2016; 44(6):2846-2858.

23. Yang W, Du WW, Li X, Yee AJ, Yang BB. Foxo3 activity promoted by non-coding effects of circular RNA and FOxo3 pseudogene in the inhibition of tumor growthand angiogenesis. Oncogene. 2016; 35(30):3919-3931.

24. Du WW, Yang W, Chen Y, Wu ZK, Foster FS, Yang Z, Li $\mathrm{X}$ and Yang BB. Foxo3 circular RNA promotes cardiac senescence by modulating multiple factors associated with stress and senescence responses. Eur Heart J. 2016.

25. LaPierre DP, Lee DY, Li SZ, Xie YZ, Zhong L, Sheng W, Deng $Z$ and Yang BB. The ability of versican to simultaneously cause apoptotic resistance and sensitivity. Cancer Res. 2007; 67(10):4742-4750. 